\title{
"Mean Field Games" as Mathematical Models for Control and Optimization of Business Activity
}

\author{
Vladimir V. Shaidurov ${ }^{a, b^{*}}$ \\ and Viktoria S. Kornienko ${ }^{\mathrm{a}, \mathrm{c}}$ \\ ${ }^{a}$ Institute of Computational Modeling SB RAS \\ 50/44 Akademgorodok, Krasnoyarsk, 660036, Russia \\ ${ }^{b}$ Tianjin University of Finance and Economics \\ 25 Zhujiang Road, Hexi District, Tianjin, 300222, China \\ 'Siberian Federal University \\ 79 Svobodny, Krasnoyarsk, 660041, Russia
}

Received 25.03.2019, received in revised form 28.03.2019, accepted 08.04.2019

The article is a review of modern mathematical economic models with the "Mean Field Games" structure. They are currently used for the predictive modelling under given control conditions or for optimizing control actions to achieve the desired result. The mathematical model is a pair of parabolic partial differential equations with a set of initial and boundary conditions for optimizing a given target functional. For them, the discretization is applied to obtain systems of nonlinear algebraic equations which are solved by computer in an iterative way to get the best instant benefit for each agent. This mathematical apparatus is used for the quantitative modelling of the distribution or the use of alternative resources, environmental problems, optimization of wages and insurance, network sales, and other economic activities to predict the aggregate behavior of the great mass of agents looking for instant personal benefit.

Keywords: mathematical economical models, Mean Field Games, Kolmogorov equation, Hamilton-Jacobi-Bellman equation, numerical solution.

This work was supported in part by Projects of the National Natural Science Foundation of China 91430108 and 11171251.

Research area: mathematical economics.

Citation: Shaydurov, V.V., Kornienko, V.S. (2019). "Mean Field Games" as mathematical models for control and optimization of business activity. J. Sib. Fed. Univ. Humanit. soc. sci., 12(4), 701-715. DOI: 10.17516/1997-1370-0418.

(c) Siberian Federal University. All rights reserved

* Corresponding author E-mail address: shaidurov04@mail.ru

ORCID: 0000-0002-7883-5804 (Shaidurov); 0000-0003-1126-2148 (Kornienko)

This work is licensed under a Creative Commons Attribution-NonCommercial 4.0 International License (CC BY-NC 4.0). 


\section{Introduction}

Mean field games (MFG for short) are a relatively new field of research developed by J.-M. Lasry and P.-L. Lions (2006a, 2006b, 2007a, 2007b). The MFG strategy helps to understand the limiting behavior of systems involving a very large number of rational agents which play differential games under partial information and symmetry assumptions. This allows economists to transfer the ideas of statistical physics to a new class of models in which the contribution of an individual player does not significantly influence on the behavior of the entire mass of players.

The appearance of this approach is caused by the great complexity of traditional approaches (for example, dynamic programming (Cormen et al., 2001)) to the study of the systems with a large number of interacting agents. It is assumed that the behavior of each player is described by some dynamic stochastic equation. The dynamics are influenced both by the position of the agent and the control action chosen by them, and by the position of other players. Each agent seeks to maximize their own benefits which also depend on their own trajectory, the control action, and the trajectories of all players.

\section{Historical review of Mean Field Game strategy}

The search for solutions of such models leads to the concept of the Nash equilibrium that goes back to the seminal works by J. Nash $(1950,1951)$.

The development of the theory continues to be relevant and interesting for researchers that leads to the emergence of new models based on the theory, which require efficient numerical algorithms. For instance, Diamond and Dybvig (1983) proposed a banking model in the form of a game played by depositors. Rochet and Vives (2004) discussed a static model of the inter-banking system. The theory of games with strategic complementarities goes back to the original works by Vives (1990) and by Milgrom and Roberts (1990). An application to games with mean field interactions can be found in the paper by Adlakha and Johari (2013). Gomes and Saude (2014) have considered Price impact models as mean field games for which the interaction between the players occurs through the distribution of the controls of the players from a perspective by partial differential equations (PDE).

The models of crowd's behavior demonstrate how the mean field game models can be versatile in the analysis of large populations. Such models are presented by the papers (Lachapelle and Wolfram, 2011) and (Achdou and Laurière, 2015) which provided numerical evidence of the explanatory power of these models. The authors 
provide a rigorous mathematical framework to try to understand complex phenomena like schooling, flocking, hurdling, etc., based on the rational behavior of individuals optimizing their own interests within a large population.

The rigorous derivation of mean-field models was considered in the original papers by Lions and Lasry (2006a, 2006b, 2007a, 2007b). Further developments using the theory of nonlinear Markov processes were obtained in (Kolokoltsov, Li, and Yang, 2011; Kolokoltsov and Yang 2013a, 2013b; Kolokoltsov, 2010) and in (Bardi and Feleqi, 2013) where methods of partial derivative equations were used. For finite state problems, the $N$-player problem was studied in (Gomes, Mohr, and Souza, 2013) where a convergence result was established. As for earlier works, the context of statistical physics and interacting particle systems were considered in (Sznitman, 1991).

There is also a growing interest in numerical methods for these problems (Lachapelle, Salomon and Turinici, 2010; Achdou and Capuzzo-Dolcetta, 2010; Achdou, 2013; Achdou, Camilli and Capuzzo-Dolcetta, 2012; Achdou and Perez, 2013; Carlini and Silva, 2013). In (Gomes, Mohr, and Souza, 2010; Ferreira and Gomes, 2013; Gueant, 2011a, 2011b) the discrete time, finite state problem, and the continuous time finite state problem were also considered. Various applications and additional models have been worked out in detail in (Gueant, 2009a, 2009b; Bardi and Feleqi, 2013; Balandat and Tomlin, 2013; Gomes and Ribeiro, 2013; Nourian et al. 2013; Tembine, 2013; Lasry, Lions, and Gueant, 2011; Lachapelle et al., 2013; Lucas and Moll, 2013; Santambrogio, 2012). Problems motivated by applications with mixed populations or with a major player were studied in (Huang, 2010, 2012). Mean field games were also analyzed using backwards-forwards stochastic differential equations in (Nguyen and Huang, 2012; Carmona and Delarue, 2013a, 2013b; Carmona and Lacker, 2013). Linear quadratic problems have been considered from distinct points of view, for instance, in (Huang, Caines, and Malhamé, 2007, 2010; Bardi and Priuli, 2013; Bensoussan et al., 2013; Li and Zhang, 2008; Cormen et al., 2001).

The approach to finding a solution considered here is based on a direct analysis of a system of two related parabolic equations: the Hamilton-Jacobi-Bellman equation, which describes the agent's optimal control problem, and the Kolmogorov equation, which describes the dynamics of the aggregated distribution of agents. The last equation is called the Fokker-Planck equation in the physical context (Carmona and Delarue, 2018). Such an approach is an advantage of MFG, since for other methods of solving problems of the control theory, the problem of finding the Nash equilibrium is reduced to solving a system of partial differential equations (Friedman, 1971) where 
the number of equations in the system is equal to a huge number of agents. This makes it almost impossible to use other optimization approaches for analyzing systems with a large number of interacting objects.

Here we focus on the discrete approximation of these equations and on an application of the MFG theory directly at discrete level. Contrary to difference schemes applied by other researches, we propose the semi-Lagrangian approximation that improves some properties of a discrete problem of this type.

\section{A brief outline of the Mean Field Game model}

A standard introduction to the mean field game theory starts with a $N$-player stochastic differential game in which the dynamics of the state $X^{i}(t)$ of $i$-th player is described by stochastic differential equation

$$
\begin{aligned}
& d X^{i}=\sigma^{i} d W^{i}-\alpha^{i} d t, \quad t \in[0, T], \\
& X^{i}(0)=x_{0}^{i} \in \mathbb{R}^{d}, \quad i=1,2, \ldots, N,
\end{aligned}
$$

where $d \geq 1 ; \sigma^{i}>0$ for all $i ; W^{i}(t)$ is an $i$-independent Brownian motion in $\mathbb{R}^{d}$. The function $\alpha^{i}(t)$ corresponds to the control function of the $i$-th player.

The aim of each player consists in the minimization of the cost functional

$$
J^{i}\left(\alpha^{1}, \ldots, \alpha^{N}\right)=\mathbb{E}\left[\int_{0}^{T} L^{i}\left(X^{i}, \alpha^{i}\right)+F\left(X^{1}, \ldots, X^{N}\right) d t+G^{i}\left(X^{i}(T)\right)\right]
$$

where $\mathbb{E}[\cdot]$ is mathematical expectation; $L^{i}, F, G$ are some Lipschitz functions.

If the above scheme can be carried out successfully, it is usually possible to prove that the optimal control found at step (2) can be used to provide approximate Nash equilibriums for the finite player game. Let us recall the definition of a Nash point: $\left(\bar{\alpha}^{1}, \ldots, \bar{\alpha}^{N}\right)$ is a Nash point if

$$
J^{i}\left(\bar{\alpha}^{1}, \ldots, \bar{\alpha}^{i-1}, \alpha^{i}, \bar{\alpha}^{i+1}, \ldots, \bar{\alpha}^{N}\right) \geq J^{i}\left(\bar{\alpha}^{1}, \ldots, \bar{\alpha}^{N}\right) \quad \forall \alpha^{i}, \forall i
$$

Such stochastic models are widely used in economic, engineering, and social science applications. The notion of Nash equilibrium is one of the most prevalent notions of equilibrium used in their analysis. However, when the number of players is large, exact Nash equilibria are notoriously difficult to identify and construct explicitly. 
So, for a large number of agents the direct solving becomes difficult to implement. To overcome these difficulties, Lasry and Lions (2006a, 2006b, 2007a) initiated the theory of mean field games for a type of games where all the players are statistically identical and only interact through their empirical distributions. These authors successfully identify the limiting problem as a couple of partial differential equations: the first one of the Hamilton-Jacobi-Bellman (HJB) type and the second one of the Kolmogorov type. Then approximate Nash equilibria for the finite-player games are derived from solutions of the limiting problem. A completely different so-called "probabilistic" approach was developed by Carmona and Delarue (2013b) where the limiting system of coupled partial differential equations is replaced by a fully coupled forward-backward stochastic differential equation. Recently, an approach based on the weak formulation of stochastic controls was introduced in (Carmona and Lacker, 2015); and the models with common noise were studied in (Carmona, Delarue and Lacker, 2016).

Here we give only the scheme of the formulation for limiting partial differential equations.

We consider an infinitely large population of agents (particles in a physical medium). The state of each agent at the instant $t \in[0, T]$ is given by a point $x(t)$ in a domain $\Omega \in \mathbb{R}^{d}$. The statistical distribution of the agents on a domain $\Omega$ is described by probability distribution $m(t, x)$. Due to uniformity of conditions, (1) is transformed into the stochastic ordinary differential equation

$$
d x=\sigma d W-\alpha d t, \quad t \in[0, T], \text { for any } x \in \Omega \text {. }
$$

Here $W(t)$ is an independent Brownian motion in $\mathbb{R}^{d} ; \sigma>0$. The vectorfunction $\alpha$ can depend on many external arguments; we simplify the description by three arguments, i.e., $\alpha(t, x, m)$.

Thus, from a stochastic point of view we get the following problem: minimize the cost functional

$$
\inf _{\alpha} \mathbb{E}\left[\int_{0}^{T} \int_{\Omega} F(t, m, \alpha) d x d t+\int_{\Omega} G(T, m) d x\right]
$$

for condition (4) at each point in $\Omega$.

The application of the "Law of large numbers" as the limiting approach (for example, accurately described in (Kloeden and Platen, 1991)) gives the following statement: 
minimize the cost functional

$$
J(\alpha)=\left[\int_{0}^{T} \int_{\Omega} F(t, m, \alpha) d x d t+\int_{\Omega} G(T, x) d x\right]
$$

with respect to $\alpha$ for the Kolmogorov equation

$$
\partial m / \partial t-\sigma^{2} \Delta m+\operatorname{div}(m \alpha)=0 \quad \text { in }[0, T] \times \Omega
$$

for the boundary condition

$$
\partial m / \partial n=0 \text { in }[0, T] \times \Gamma
$$

and the initial condition

$$
m(0, x)=m_{0}(x) \quad \text { on } \Omega .
$$

Here $\Delta m=\sum_{1 \leq i \leq d} \partial^{2} m / \partial x_{i}^{2}$ is the Laplace operator and $\partial m / \partial n$ is the outwardpointing derivative.

In other context (especially in the physical one), the Kolmogorov equation is known as the Fokker-Plank equation). Boundary condition (8) prevents the loss of density $m$ with time.

Now we briefly describe only a formal way to get the optimality conditions for this differential problem. The rigorous derivation can be found in (Bensoussan, Frehse and Yam, 2013) under some general concavity conditions. We will not use these differential justifications in our description and give them only as the tip.

Multiply (7) by an arbitrary relatively smooth function $v(t, x)$ and integrate by parts with respect to $t$ and $x$ :

$$
\begin{gathered}
-\int_{0}^{T} \int_{\Omega}\left(\partial v / \partial t+\sigma^{2} \Delta v+\alpha \cdot \nabla v\right) m \mathrm{~d} \Omega \mathrm{d} t \\
+\int_{\Omega}\left(v(T, x) m(T, x)-v(0, x) m_{0}(x)\right) \mathrm{d} \Omega=0
\end{gathered}
$$

taking into account the following boundary conditions similar to (8): 


$$
\partial v / \partial n=0 \quad \text { on }[0, T] \times \Gamma \text {. }
$$

In addition to the cost functional, we also introduce the Lagrangian $\mathfrak{I}(m, \alpha, v)$ for the problem (6)-(9):

$$
\begin{aligned}
& \mathfrak{I}(m, \alpha, v):=J(m, \alpha)+\int_{0}^{T} \int_{\Omega}\left(\partial v / \partial t+\sigma^{2} \Delta v+\alpha \cdot \nabla v\right) m \mathrm{~d} \Omega \mathrm{d} t \\
& -\int_{\Omega}\left(v(T, x) m(T, x)-v(0, x) m_{0}(x)\right) \mathrm{d} \Omega .
\end{aligned}
$$

Thus, the minimization problem (6)-(9) can be rewritten (Bensoussan, Frehse and Yam, 2013) as the saddle point problem

$$
\inf _{(m, \alpha)} \sup _{v} \mathfrak{I}(m, \alpha, v)
$$

After "differentiation" with respect to some functions, we get the "backward" Hamilton-Jacobi-Bellman equation with the initial and boundary conditions:

$$
\begin{gathered}
\partial v / \partial t+\sigma^{2} \Delta v+\alpha \cdot \nabla v=-\partial F / \partial m \text { on }[0, T] \times \Omega, \\
v(T, x)=G(T, x) \quad \text { on } \bar{\Omega}, \\
\partial v / \partial n=0 \quad \text { on }[0, T] \times \Gamma, \\
\partial F / \partial \alpha_{i}=-m \partial v / \partial x_{i} \text { on }[0, T] \times \bar{\Omega} .
\end{gathered}
$$

This initial-boundary problem characterizes a saddle point in addition to (6)-(9).

\section{The numerical solution of the saddle-point problem}

The first step of the numerical solution of problem (13) consists in the difference approximation of the Kolmogorov and Hamilton-Jacobi-Bellman problems. The standard widespread approximations provide monotone schemes for both problems and give the total task for the minimization of each $\alpha_{i}$ over all domain $\bar{\Omega}$. Last time, the implementation of special approximations (Shaidurov, Efremov and Gileva, 2018; Shaidurov, Viatkin, Kuchunova, 2018) for these problems led to the disintegration of the total minimization to local pointwise ones (Lachapelle, Salomon and Turinici, 
2010; Shaydurov, Zhang and Kornienko, 2019). Therefore, the discretization of the above differential problem looks as follows.

First, the special difference approximation of the Kolmogorov problem is constructed in the form of the system of linear algebraic equations

$$
A M=B
$$

for the vector $M=\left\{m\left(t_{j}, x_{s}\right)\right\}_{s=1, \ldots, N}^{j=1, \ldots, K}$ defined from one time level $t_{j}$ to the next one $t_{j+1}$. One of the main properties of this approximation is to preserve the integral of $m(t, x)$ over $\Omega$ for each time step $t_{j}$ at a discrete level.

Second, we take the approximation

$$
J^{h}(m, \alpha, v)
$$

of cost functional (6) by an appropriate quadrature rule.

Third, the approximation of the Hamilton-Jacobi-Bellman equations is performed by another different scheme to get the system of algebraic equations

$$
A^{T} V=C
$$

with the transposed matrix for the vector $V=\left\{v\left(t_{j}, x_{s}\right)\right\}_{s=1, \ldots, N}^{j=0, \ldots, K-1}$ defined from one time level $t_{j+1}$ to the previous one $t_{j}$ in backward order to ensure the stability of computations.

Finally, we need some approximation of (17)

$$
\mathbb{D}_{i}\left(A_{i}\right)=S_{i}, i=1, \ldots, d,
$$

for the vectors $A_{i}=\left\{\alpha_{i}\left(t_{j}, x_{s}\right)\right\}_{s=1, \ldots, N}^{j=0, \ldots, K-1}$ defined independently at any point $\left(t_{j}, x_{s}\right)$. To ensure the unique solvability of $(17), \mathbb{D}_{i}\left(A_{i}\right)$ must be monotone.

As a result, the computational algorithm at discrete level looks like this.

Suppose that some initial approximations $\alpha_{i}^{h}\left(t_{j}, x_{s}\right)$ and $m^{h}\left(t_{j}, x_{s}\right)$ are given. For example, we can take $\alpha_{i}^{h}\left(t_{j}, x_{s}\right)=0$ for all $\left(t_{j}, x_{s}\right)$ and compute $m^{h}\left(t_{j}, x_{s}\right)$ from 
(18). Better approximations of the control grid functions $\alpha_{i}^{h}\left(t_{j}, x_{s}\right)$ is computed in the following iterative way.

\section{Iterative algorithm}

1. Solve (20) to get $v^{h}\left(t_{j}, x_{s}\right)$.

2. Compute $\tilde{\alpha}_{i}^{h}\left(t_{j}, x_{s}\right)$ by (21).

3. Compute $\tilde{m}^{h}\left(t_{j}, x_{s}\right)$ by (18).

4. Compute $J^{h}\left(\tilde{m}^{h}, \tilde{\alpha}^{h}\right)$ by (19).

5. If $\left|J^{h}\left(m^{h}, \alpha^{h}\right)-J^{h}\left(\tilde{m}^{h}, \tilde{\alpha}^{h}\right)\right|>$ Tol then $\left\{\alpha^{h}:=\tilde{\alpha}^{h} ; m^{h}:=\tilde{m}^{h}\right.$; go to 1$\}$.

6. Take $\tilde{\alpha}^{h}$ and $\tilde{m}^{h}$ as an approximate solution of (13).

In many cases it can be showed that (21) ensures the steepest descent of the above estimate for the difference between values of the discrete cost functional.

\section{Conclusion}

The origin of the Mean Field Games methodology is related to particle physics. This theory has proven to be very effective for handling with a huge number of particles to describe the dynamics or the equilibrium of the averaged state of particles, taking into account the interparticle interaction. Introducing one or several "mean fields" as the medium of interaction of particles, in many cases it is possible to fairly accurately describe the aggregative behavior of particles, despite the negligible contribution of each particle to this behavior. The transition to economic models is due to the possibility of replacing particles with agents that interact in a socioeconomic or strategic environment. Note that this approach is not a new wave of econophysics, modifying physical laws in socio-economic systems by analogy. In MFG, the mathematical apparatus developed in particle physics is used with a new economic and social content of the formal parameters of the models.

The difference of Mean Field Games from the usual game theory with $N$ players is the unification of inter-individual interaction strategies. The transition to the Mean Field Game with a huge $N$ can only be made under the condition that the other players are considered to be representatives of the "crowd" that influences the decision-making and does not directly influence individuals. Obviously, in real-life tasks, information from the nearest neighbours-agents (tenants or colleagues) is also taken into account, but it is summarized with data from the mass media and other sources about the behavior of the "crowd" and allows the agent to generate more optimal individual behavior. Such a strong limitation as the unification of interindividual interactions, in 
newer models can be weakened by greater freedom of choice of the control function $F$ depending on time, coordinates and density with the possibility of limited resources and discontinuous behavior.

In simple economic models, agents weakly relate to each other: they are guided by their own interest and market prices. In other words, prices are mediators of social interactions. Then the theory of Mean Field Games works well if each agent can put himself in the place of another agent whose behavior is predicted by this theory. If other concepts such as traditions, social values, etc., are included in the reasoning, it is possible to identify their statistical nature and include them in the model as additional "mean fields". The mathematical theory of the Mean Field Games permits the location of the agent at several coordinates with the "crowd" interaction inside several Mean Fields.

So, rapidly progressing models of the Mean Field Games due to more complex statements offer the great possibilities of predictive modelling in the field of economics and management.

\section{References}

Achdou, Y. (2013). Finite Difference Methods for Mean Field Games. In HamiltonJacobi Equations: Approximations, Numerical Analysis and Applications. Lecture Notes in Mathematics, 2074, 1-47. DOI: 10.1007/978-3-642-36433-4_1

Achdou, Y., Camilli, F. \& Capuzzo-Dolcetta, I. (2012). Mean field games: numerical methods for the planning problem. In SIAM J. Control Optim., 50(1), 77-109. DOI: 10.1137/100790069

Achdou, Y., Capuzzo-Dolcetta, I. (2010). Mean field games: numerical methods. In SIAM J. Numer. Anal., 48(3), 1136-1162. DOI: 10.1137/090758477

Achdou, Y., Laurière, M. (2015). On the system of partial differential equations arising in mean field type control. In Discrete and Continuous Dynamical Systems, A, 35, 3879-3900. DOI: 10.3934/dcds.2015.35.3879

Achdou, Y., Perez, V. (2012). Iterative strategies for solving linearized discrete mean field games systems. In Netw. Heterog. Media, 7(2), 197-217. DOI: 10.3934/ nhm.2012.7.197

Adlakha, S., Johari, R. (2013). Mean field equilibrium in dynamic games with strategic complementarities. In Operations Research, 61, 971-989. DOI: 10.1287/ opre.2013.1192

Balandat, M., Tomlin, C. (2013). On efficiency in mean field differential games. In 2013 ACC Paper Abstract, 2527-2532. DOI: 10.1109/ACC.2013.6580214 
Bardi, M., Feleqi, E. (2013). The derivation of ergodic mean field game equations for several populations of players. In Dyn. Games Appl., 3, 523. DOI: 10.1007/s13235013-0088-5

Bardi, M., Feleqi, E. (2016). Nonlinear elliptic systems and mean field games. In Nonlinear Differ. Equ. Appl., 23, 23-44. DOI: 10.1007/s00030-016-0397-7

Bardi, M., Priuli, F. (2013). LQG mean-field games with ergodic cost. In 52nd IEEE Conference on Decision and Control, Florence, 10-13 Dec. DOI: 2013.10.1109/ CDC.2013.6760255

Bensoussan, A., Frehse, J. \& Yam, P. (2013). Mean Field Games and Mean Field Type Control Theory, Berlin, Springer, 128 p.

Bensoussan, A., Sung, K., Yam, S. \& Yung, S. (2013). Linear-quadratic mean field games. In Dyn. Games Appl., 3(4), 537-552. DOI: 10.1007/s13235-013-0090-y

Carlini, E., Silva, F.J. (2013). A fully-discrete semi-Lagrangian scheme for a first order mean field game problem. In SIAM J. Numer. Anal., 52(1), 45-67. DOI: $10.1137 / 120902987$

Carmona, R., Lacker, D. (2015). A probabilistic weak formulation of mean field games and applications. In Ann. Appl. Probab., 25 (3), 1189-1231.DOI: 10.1214/14AAP1020

Carmona, R., Delarue, F. (2013a). Mean field forward-backward stochastic differential equations. In Electron. Commun. Probab., 18 (68), 1-15. DOI: 10.1214/ ECP.v18-244

Carmona, R., Delarue, F. (2013b). Probabilistic analysis of mean-field games. In SIAM J. Control Optim., 51(4), 2705-2734. DOI: 10.1137/120883499

Carmona, R., Delarue, F. \& Lacker, D. (2016). Mean field games with common noise. In Ann. Probab., 44 (6), 3740-30803.

Carmona, R., Delarue, F. (2018). Probabilistic Theory of Mean Field Games with Applications I. Mean Field FBSDEs, Control and Games. Springer, Berlin, 721 p.

Cormen, T.H., Leiserson, C.E., Rivest, R.L. \& Stein, C. (2001). Introduction to Algorithms (2nd ed.), Cambridge, Massachusetts, MIT Press, 344 p.

Diamond, D.W., Dybvig, P.H. (1983). Bank runs, deposit insurance, and liquidity. In The Journal of Political Economy, 91, 401-419.

Ferreira, R., Gomes, D. (2014). On the convergence of finite state mean-field games through $\Gamma$-convergence. In Journal of Mathematical Analysis and Applications, 418 (1), 211-230. DOI: 10.1016/j.jmaa.2014.02.044

Friedman, A. (1971). Differential games. Wiley, New York. 370 p. 
Gomes, D., Mohr, J., \& Souza, R. (2010). Discrete time, finite state space mean field games. In J. Math. Pures. Appl. 93(2), 308-328. DOI: 10.1016/j.matpur.2009.10.010

Gomes, D., Ribeiro, R. (2013). Mean field games with logistic population dynamics. In 52nd IEEE conference on decision and control, Florence, December 2013, 14158267, DOI: 10.1109/CDC.2013.6760258

Gomes, D.A., Saude, J. (2014). Mean field games models - a brief survey. In Dynamic Games and Applications, 4, 110-154. DOI: 10.1007/s13235-013-0099-2

Gueant, O. (2009a). A reference case for mean field games models. In J. Math. Pures. Appl., 92(3), 276-294, DOI: 10.1016/j.matpur.2009.04.008

Gueant, O. (2009b). Mean field games and applications to economics. Ph.D. Thesis, Université Paris Dauphine, Paris, 98 p.

Gueant, O. (2011a). From infinity to one: the reduction of some mean field games to a global control problem. Available at: https://arxiv.org/abs/1110.3441

Gueant, O. (2011b). An existence and uniqueness result for mean field games with congestion effect on graphs. In Appl. Math. Optim., 72, 291. DOI: 10.1007/s00245-014-9280-2

Huang, M. (2010). Large-population LQG games involving a major player: the Nash certainty equivalence principle. In SIAM J. Control Optim., 48(5), 3318-3353. DOI: $10.1137 / 080735370$

Huang, M. (2012). Mean field stochastic games with discrete states and mixed players. In Proc. Game Nets, Lecture Notes of the Institute for Computer Sciences, Social Informatics and Telecommunications Engineering, 105, 138-151. DOI: 10.1007/978-3-642-35582-0_11

Huang, M., Caines, P.E. \& Malhamé, R.P. (2007). Large-Population Cost-Coupled LQG Problems with Nonuniform Agents: Individual-Mass Behavior and Decentralized E-Nash Equilibria, In IEEE Trans. Automat. Contr., 52, 1560-1571. DOI: 10.1109/ TAC.2007.904450

Huang, M., Caines, P., \& Malhamé, R.P. (2010). The NCE (mean field) principle with locality dependent cost interactions. In IEEE Trans. Autom. Control, 55(12), 2799-2805. DOI: 10.1109/TAC.2010.2069410

Kloeden, P.E., Platen, E. (1992). Numerical Solution of Stochastic Differential Equations, Springer, Heidelberg.

Kolokoltsov, V. (2010). Nonlinear Markov processes and kinetic equations. Cambridge, Massachusetts. MIT Press, 394 p.

Kolokoltsov, V., Li, J.J., Yang, W. (2011). Mean field games and nonlinear Markov processes. Available at: https://arxiv.org/abs/1112.3744v2 
Kolokoltsov, V., Yang, W. (2013a). Existence of solutions to path-dependent kinetic equations and related forward-backward systems. In Open Journal of Optimization, 2, 39-44. DOI: 10.4236/ojop.2013.22006

Kolokoltsov, V., Yang, W. (2013b). Sensitivity analysis for HJB equations with an application to a coupled backward-forward system. Available at: https://arxiv.org/ abs/1303.6234

Lachapelle, A., Lasry, J.-M., Lehalle, C.-A. \& Lions, P.-L. (2016). Efficiency of the price formation process in presence of high frequency participants: a mean field game analysis. In Math. Finan. Econ., 10, 223. DOI: 10.1007/s11579-0150157-1

Lachapelle, A., Salomon, J., Turinici, G. (2010). Computation of mean field equilibria in economics. In Mathematical Models and Methods in Applied Sciences, 20(4), 467-588. DOI: 10.1142/S0218202510004349

Lachapelle, A., Wolfram, M.T. (2011). On a mean field game approach modeling congestion and a version in pedestrian crowds. In Transportation Research Part B: Methodological, 45, 1572-1589. DOI: 10.1016/j.trb.2011.07.011

Lasry, J.-M., Lions, P.-L. (2006a). Mean field games. I. The stationary case. [Jeux ’a champ moyen. I. Le casstationnaire]. In C.R. Math. Acad. Sci. Paris, 343(9), 619625. DOI: 10.1016/j.crma.2006.09.019

Lasry, J.-M., Lions, P.-L. (2006b). Mean field games. II. Finite horizon and optimal control. [Jeux à champ moyen. II. Horizon fini et contrôle optimal]. In C.R. Math. Acad. Sci. Paris, 343(10), 679-684. DOI: 10.1016/j.crma.2006.09.018

Lasry, J.-M., Lions, P.-L. (2007a). Mean field games. In Cahiers de la Chaire Finance et Développement Durable [Papers of Chair of Finance and Sustainable Development], 2, 205-266.

Lasry, J.-M., Lions, P.-L. (2007b). Mean field games. In Jpn. J. Math., 2(1), 229260. DOI: $10.1007 / \mathrm{s} 11537-007-0657-8$

Lasry, J.-M., Lions, P.-L., \& Gueant, O. (2011). Mean field games and oil production. In The Economics of Sustainable Development, 1, available at: https://ideas.repec. org/p/hal/journl/hal-01393104.html

Li, T., Zhang, J.-F. (2008). Asymptotically optimal decentralized control for large population stochastic multiagent systems. In IEEE Trans Autom Control, 53(7), 16431660. DOI: 10.1109/TAC.2008.929370

Lucas, R.E., Moll, B. (2014). Knowledge growth and the allocation of time. In J. Polit. Econ., 122 (1), 1-51. DOI: 10.1086/674363 
Milgrom, P., Roberts, J. (1990). Rationalizability, learning, and equilibrium in games with strategic complementarities. In Econometrica, 58, 1255-1277. DOI: $10.2307 / 2938316$

Nash, J. (1950). Equilibrium points in n-person games. Proceedings of the National Academy of Sciences of the USA, 36, 48-49.

Nash, J. (1951). Non-cooperative games. In Annals of Mathematics, 54, 286-295.

Nguyen, S.L., Huang, M. (2012). Linear-quadratic-Gaussian mixed games with continuum-parametrized minor players. In SIAM J. Control Optim. 50(5), 2907-2937. DOI: $10.1137 / 110841217$

Nourian, M., Caines, P., Malhamé, R.P. \& Huang, M. (2013). Nash, social and centralized solutions to consensus problems via mean field control theory. In IEEE Trans. Autom. Control, 58(3), 639-653. DOI: 10.1109/TAC.2012.2215399

Rochet, J.C., Vives, X. (2004). Coordination failures and the lender of last resort. In Journal of the European Economic Association, 2, DOI: 1116$1148.10 .1162 / 1542476042813850$

Santambrogio, F. (2012). A modest proposal for MFG with density constraints. In Netw. Heterog. Media, 7(2), 337-347. DOI: 10.3934/nhm.2012.7.337

Shaidurov, V., Efremov, A., Gileva, L. (2018). Semi-Lagrangian difference approximations for distinct transfer operators. In AIP Conference Proceedings, 2025, 020004. DOI: 10.1063/1.5064877

Shaidurov, V., Viatkin, A., Kuchunova, E. (2018). Semi-Lagrangian difference approximations with different stability requirements. In Russian J. Numer. Anal. Math. Modelling, 33(2), 123-135. DOI: 10.1515/rnam-2018-0011

Shaydurov, V., Zhang, S., Kornienko, V. (2019). Computation of Mean-Field Equilibria with Correlated Stochastic Processes. In Lecture Notes in Computer Science. 11386, 468-475.

Sznitman, A.-S. (1991). Topics in propagation of chaos. In Lecture notes in math., 1464, 165-251. DOI: 10.1007/BFb0085169

Tembine, H. (2014). Energy-constrained mean field games in wireless networks. In J. Strateg. Behav. Environ., 4(2), 187-211, DOI: 10.1561/102.00000040

Vives, X. (1990). Nash equilibrium with strategic complementarities. In J. of Mathematical Economics, (19), 305-321. DOI: 10.1016/0304-4068(90)90005-T 


\title{
«Игры среднего поля»
}

как математические модели управления

и оптимизации экономической активности

\author{
В.В. Шайдуров ${ }^{\text {a, }}$, В.С. Корниенко ${ }^{\mathrm{a}, \mathrm{s}}$ \\ ${ }^{a}$ Институт вычислительного моделирования РАН \\ Россия, 660036, Красноярск, Академгородок, 50/44 \\ ${ }^{6}$ Тяньизиньский университет финансов и экономики \\ Китай, Тяньизинь, Хэси \\ ${ }^{8}$ Сибирский федеральный университет \\ Россия, 660041, Красноярск, пр. Свободный, 79
}

Статья посвящена обзору современных математических экономических моделей в терминах теории "Mean Field Game". В настоямее время такие модели используются для прогностического моделирования при заданных условиях управления или для поиска оптимального управления динамической системой для достижения желаемого результата. Математическая модель представляет собой пару параболических уравнений в частных производных с начальнылми и граничными условиями для оптимизации заданного иелевого функционала. Для них применяется дискретизаџия с иелью формирования системы нелинейных алгебраических уравнений, которые решаются на компьютере итерационным образом для получения наибольшего текущего выигрыша каждым агентом. Данный математический аппарат используется для количественного моделирования распределения или формирования альтернативных ресурсов, решения экологических проблем, оптимизачии заработной платы и страхования, сетевых продаж и других видов экономической деятельности для предсказания агрегатного поведения огромной массы агентов, ищущих собственную выгоду.

Ключевые слова: математические экономические модели, игры среднего поля, уравнение Колмогорова, уравнение Гамильтона-Якоби-Беллмана, численное решение.

Эта работа была частично поддержана Проектами Государственного фонда естественных наук Китая 91430108 и 11171251.

Научная специиальность: 06.18.00 - математические методы в экономике. 